\title{
Thermoelektrischer Kohlenwasserstoffsensor in Dickschichttechnik mit Pt | PtRh Thermopile zur On-Board- Diagnose eines Diesel-Oxidations-Katalysator
}

\author{
Sven Wiegärtner ${ }^{1}$, Gunter Hagen ${ }^{1}$, Jaroslaw Kita ${ }^{1}$, Daniela Schönauer-Kamin ${ }^{1}$, Willibald Reitmeier ${ }^{2}$, \\ Katharina Burger ${ }^{2}$, Philippe Grass ${ }^{2}$, Marcel Kaspar ${ }^{3}$, Hans-Peter Rabl ${ }^{3}$, Alistair Prince ${ }^{4}$, Peter \\ Weigand $^{5}$, Ralf Moos ${ }^{1}$ \\ ${ }^{1}$ Lehrstuhl für Funktionsmaterialien, Universität Bayreuth, Universitätsstr. 30, 95447 Bayreuth, \\ Deutschland, Tel.: +49921557401, E-Mail: funktionsmaterialien@uni-bayreuth.de; \\ ${ }^{2}$ Continental Automotive GmbH, Div.Powertrain, Siemensstraße 12, 93055 Regensburg, Deutschland; \\ ${ }^{3}$ OTH Regensburg, Fakultät Maschinenbau, Galgenbergstr. 30, 93053 Regensburg, Deutschland; \\ ${ }^{4}$ DuPont (U.K.) Limited, Coldharbour Lane, Frenchay, Bristol, BS16 1QD, England; \\ ${ }^{5}$ DuPont de Nemours GmbH, COB, Hugenottenallee 175, 63263 Neu-Isenburg, Deutschland;
}

\begin{abstract}
Kurzfassung
Kohlenwasserstoffsensoren können zur vorgeschriebenen On-Board-Diagnose eines DieselOxidations-Katalysators eingesetzt werden. Ein möglicher Sensortyp zur Detektion unverbrannter Kohlenwasserstoffe im automobilen Abgas nutzt das thermoelektrische Prinzip. Dabei entsteht zwischen zwei unterschiedlichen Materialien A und B, die in Dickschichttechnik auf einem elektrisch isolierenden Substrat aufgebracht werden, eine Thermospannung, die die Messgröße ist. Eine Seite der Kontaktstelle der beiden Materialien wird mit einer inerten Schicht und die andere Seite mit einer katalytisch hochaktiven Schicht bedeckt. Aufgrund der Oxidationsreaktion von Kohlenwasserstoffen und der daraus resultierenden Temperaturerhöhung an der katalytisch aktiven Schicht entsteht eine Thermospannung zwischen den beiden Kontaktstellen. Um das Messsignal und somit die Empfindlichkeit des Sensors zu erhöhen, kann der Betrag der Thermospannung durch Aneinanderreihung mehrerer Thermoelementpaare zum sog. "Thermopile" erhöht werden. Aufgrund der hohen Einsatztemperaturen und der rauen Atmosphäre im Rohabgas eines Automobils werden in dieser Arbeit unter anderen die Edelmetalle Platin und Platin mit $10 \mathrm{wt} \%$ Rh als Materialien A und B gewählt. Hierbei handelt es sich um die Materialpaarung eines standardisierten Thermoelements vom Typ S. Die katalytisch aktive Schicht besteht aus einem porösen $\mathrm{Al}_{2} \mathrm{O}_{3}$, welches mit 1 wt $\%$ Pt versetzt wird. Zusätzlich wird auf der Rückseite des Sensorelements ein Platinheizer angebracht, so dass eine konstante Sensorbetriebstemperatur von bis zu $650^{\circ} \mathrm{C}$ bereitgestellt werden kann.
\end{abstract}

Keywords: Kohlenwasserstoffsensor, Abgassensor, Hochtemperatur-Anwendungen, Dickschichttechnologie, Pt|PtRh-Thermoelement

\section{Hintergrund}

Diesel-Oxidations-Katalysatoren (DOC) im automobilen Abgasstrang haben zum einen die Aufgabe, unverbrannte Kohlenwasserstoffe $\left(\mathrm{C}_{\mathrm{x}} \mathrm{H}_{\mathrm{y}}\right)$ und Kohlenmonoxid (CO) zu Kohlendioxid $\left(\mathrm{CO}_{2}\right)$ und Wasser $\left(\mathrm{H}_{2} \mathrm{O}\right)$ umzusetzen [1]. Eine weitere Reaktion, die im DieselOxidations-Katalysator abläuft und die die weitere Umsetzung des Abgases begünstigt, ist der Umsatz von Stickstoffmonoxid (NO) zu Stickstoffdioxid $\left(\mathrm{NO}_{2}\right)$. Damit ist aufgrund des hochreaktiven $\mathrm{NO}_{2}$ bei Harnstoff-SCRSystemen die Stickoxidreduktion mit Ammoniak zu Stickstoff $\left(\mathrm{N}_{2}\right)$ und $\mathrm{H}_{2} \mathrm{O}$ bei tiefen Temperaturen begünstigt [2]. Wird der DieselOxidations-Katalysator vor einem DieselPartikelfilter (DPF) angeordnet, fördert das gebildete $\mathrm{NO}_{2}$ auch noch die Niedertemperatur-Rußoxidation [3]. Im Falle nacheingespritzter Kohlenwasserstoffe erhöht der $\mathrm{C}_{x} \mathrm{H}_{y}$-Umsatz die Abgastemperatur [4], was zum Beispiel den Rußabbrand im Partikelfilter begünstigt. Somit ist der Diesel-OxidationsKatalysator ein emissionsrelevantes Bauteil, welches im Rahmen der vorgeschriebenen OnBoard-Diagnose (OBD) mithilfe eines Sensors überwacht werden muss [5].

Eine Möglichkeit, eine nachlassende Konversionsleistung von Oxidationskatalysatoren zu detektieren, liegt darin, die Kohlenwasserstoffkonzentration im Abgas nach Katalysator mit Hilfe von Kohlenwasserstoffsensoren zu messen. Bei einem defekten, gealterten oder auch bei einem noch kalten 
Katalysator detektieren die Sensoren die nicht umgesetzten Kohlenwasserstoffe [6, 7].

\section{Sensoraufbau}

Wie in Abb. 1 links zu sehen, werden zwei Elektroden des Materials $A$ auf ein isolierendes Substrat, aufgebracht. Mit einem Material B werden diese beiden Elektroden verbunden. Die eine Kontaktstelle Material A-B wird mit einer inerten Schicht abgedeckt (s. Abb. 1). Die andere Kontaktstelle Material B-A wird mit einer katalytisch aktiven Schicht bedruckt. Dort können die Kohlenwasserstoffe oxidiert und Reaktionswärme freigesetzt werden. Die inertisierte Seite hingegen bleibt von der Kohlenwasserstoffzugabe unberührt. Somit bildet sich über den Kontaktstellen eine Temperaturdifferenz aus. Die Spannung $U_{\text {th }}$, die über die beiden Elektroden des Materials A abgegriffen werden kann, ist direkt proportional zu dieser Temperaturdifferenz zwischen den beiden Seiten und hängt somit monoton von der Konzentration der Kohlenwasserstoffe ab. Die Spannung $U_{\text {th }}$ berechnet sich nach GI. 1 aus dem Seebeck-Koeffizienten $k_{\mathrm{AB}}$ der beiden Materialien $A$ und $B$ und den beiden Temperaturen $T_{\text {KAT, heiß }}$ und $T_{\text {inert }}$.

$U_{\text {th }}=k_{\mathrm{AB}} \cdot\left(T_{\mathrm{KAT}, \text { heiß }}-T_{\text {inert }}\right)$

Wie in Abb. 1 rechts dargestellt, kann der Betrag der Thermospannung durch Aneinanderreihung mehrerer Thermoelementpaare zum sog. „Thermosäule" erhöht werden, siehe z.B. [8]. Die dann erhaltene Thermospannung multipliziert sich mit der Anzahl $n$ an Thermoelementpaaren gemäß GI. 2 .

$U_{\text {th }}=n \cdot k_{\mathrm{AB}} \cdot\left(T_{\text {KAT, heiß }}-T_{\text {inert }}\right)$

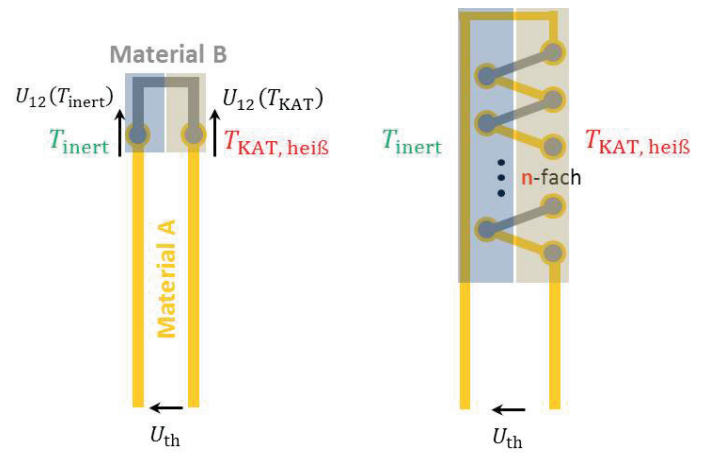

$U_{\text {th }}=k_{12}\left(T_{\text {KAT }}-T_{\text {inert }}\right) \quad U_{\text {th }}=\mathrm{n} \cdot k_{12}\left(T_{\text {KAT }}-T_{\text {inert }}\right)$

Abb. 1: Prinzip und Aufbau des thermoelektrischen Kohlenwasserstoffsensors

Die absolute Sensorbetriebstemperatur wird durch den rückseitig aufgebrachten Heizer bereitgestellt. Diese muss idealerweise so hoch sein, dass alle Kohlenwasserstoffe am Katalysator reagieren.
Aufgrund der hohen Einsatztemperaturen und der rauen Abgasatmosphäre wurden in dieser Arbeit auch (in Ergänzung zu [9]) Sensoren auf $\mathrm{Al}_{2} \mathrm{O}_{3}$-Substraten mit Pt- und Pt|RhElektroden hergestellt. Für diese Pt-Elektrode wurde die Paste 9142 (A013) und für die $\mathrm{Pt} \mid \mathrm{Rh}$-Elektrode die Paste 9152 (D101679146C) von DuPont verwendet. Dadurch werden die Sensoren hochtemperaturstabiler und sind somit abgastauglich. Ein weiterer Vorteil ist es, dass die Materialpaarung Pt | PtRh ein genormtes Thermoelement (Typ S) darstellt und somit bekannte Kennlinien anwendbar sind.

Die Thermosäule ist auf der einen Seite mit einer Dielektrikumspaste inertisiert. Die aktive Seite wurde mit porösem $\mathrm{Al}_{2} \mathrm{O}_{3}$, welches mit $1 \mathrm{wt} \% \mathrm{Pt}$ versetzt wurde (siehe z.B. [10]), katalytisch beschichtet.

Das Heizelement auf der Rückseite des Sensorsubstrates ist ein siebgedrukter Platinmäander, der in 4-Leiter-Technik kontaktiert wird. Mithilfe des bekannten temperaturabhängigen Widerstands des Platinmäanders lässt sich somit eine genaue Sensortemperatur einstellen.

\section{Labormessungen}

Im Zuge dieser Arbeit wurden Sensoren mit 15 Thermopaaren in einer Sensortestanlage mit

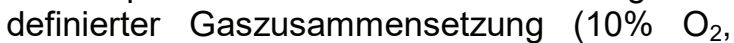
Kohlenwasserstoffe, Rest $\mathrm{N}_{2}$ ) getestet, um die theoretische Funktionsweise der Sensoren nachzuweisen und die Sensorkennlinien bezüglich Propen $\left(\mathrm{C}_{3} \mathrm{H}_{6}\right)$ zu ermitteln. Dabei wurden die Sensoren auf $600{ }^{\circ} \mathrm{C}$ beheizt und dann mit Testgas beaufschlagt $(0-2350$ ppm $\mathrm{C}_{3} \mathrm{H}_{6}$ ). Es kamen Sensoren auf LTCC Substraten mit $\mathrm{Au}$ und $\mathrm{Pt}$ als Elektrodenmaterialien, Sensoren auf $\mathrm{Al}_{2} \mathrm{O}_{3}$-Substraten mit $\mathrm{Au}$ und $\mathrm{Pt}$ als Elektroden-materialien, sowie die erwähnten hoch-temperaturstabilen Sensoren auf $\mathrm{Al}_{2} \mathrm{O}_{3}$ Substraten mit Pt | PtRhThermoelementen zur Anwendung. Wie in Abb. 2 (oben) zu sehen ist, nimmt die gemessene Sensorspannung in der o.g. Reihenfolge der Sensoren ab. Dies entspricht den theoretischen Erwartungen, da zum einen die Wärmeleit-fähigkeit des $\mathrm{Al}_{2} \mathrm{O}_{3}$-Substrates höher ist als das des LTCC-Substrates und somit der messbare Temperaturunterschied aufgrund der exothermen Reaktion zwischen den beiden Kontaktstellen geringer ist. Zum zweiten ist der Seebeck-Koeffizient der Materialpaarung $\mathrm{Pt} \mid \mathrm{PtRh}$ geringer als der der $\mathrm{Au}$ | Pt-Paarung. Rechnet man diese Spannung in den Temperaturunterschied 
zwischen den Kontaktstellen um und berücksichtigt den jeweiligen temperaturabhängigen Seebeck-Koeffizienten der Materialpaarung, so liegen die Kennlinien der Sensoren auf $\mathrm{Al}_{2} \mathrm{O}_{3}$-Substraten wieder aufeinander (s. Abb. 2, unten). Die theoretischen Erwartungen sind somit bestätigt.
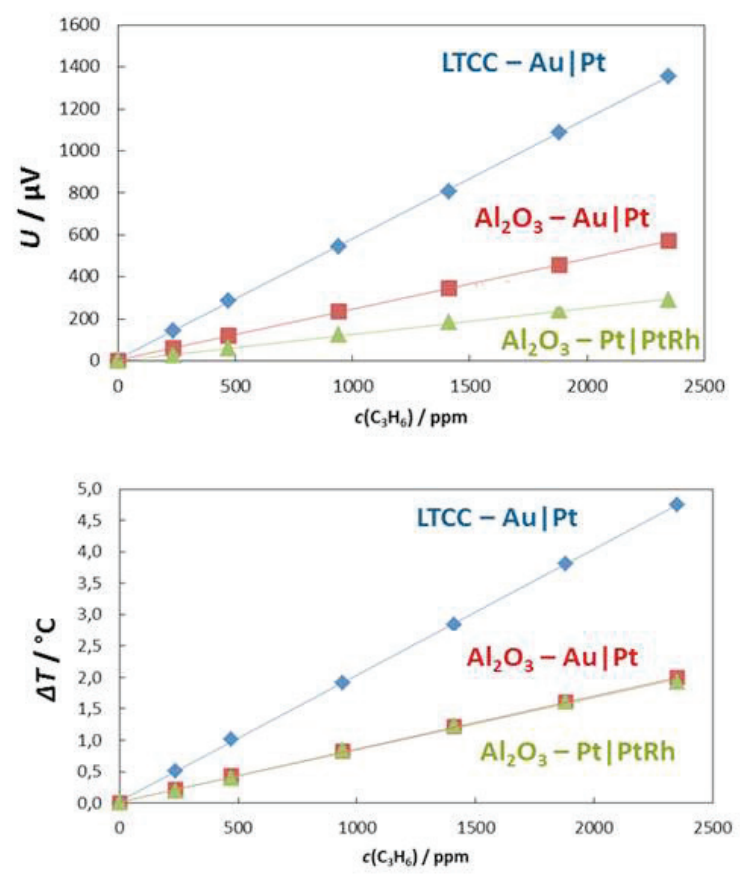

Abb. 2: Offsetkorrigierte Kennlinien getesteter Sensoren; oben: gemessene Spannung, unten: Spannung mit den jeweiligen Seebeck-Koeffizienten umgerechnet in den Temperaturunterschied zwischen inerter und katalytisch aktivierter Seite

\section{Messungen am Motorprüfstand}

Aufgrund der guten Hochtemperaturstabilität der Sensoren auf $\mathrm{Al}_{2} \mathrm{O}_{3}$-Substraten mit den $\mathrm{Pt} \mid \mathrm{PtRh}-T h e r m o p a a r e n$ konnten auch erste vielversprechende Ergebnisse im Abgas eines Motorprüfstandes erzielt werden. Bei dieser Messung wurde ein Sensor vor dem DieselOxidations-Katalysator eines handelsüblichen Abgasstranges und ein weiterer nach diesem direkt im Abgasstrang (Hauptstrom) des Motorprüfstandes platziert, wie schematisch in Abb. 3. gezeigt.

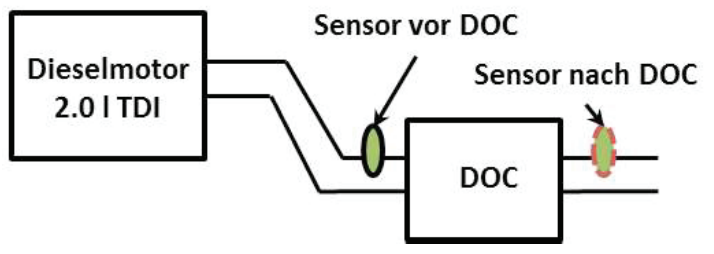

Abb. 3: Schematischer Aufbau der Messung am Motorprüfstand
Der Motor wurde bei konstanter Drehzahl und Last betrieben. Mittels gezielter Nacheinspritzungen wurde der Kohlenwasserstoffgehalt im Abgas kurzzeitig erhöht: Zunächst wurde 2, 3, 4 und 5 Sekunden lang mit $5 \mathrm{mg} / \mathrm{Hub}$ nacheingespritzt, dann 4 und 5 Sekunden lang mit $8 \mathrm{mg} / \mathrm{Hub}$ und am Ende noch 2, 3, 4 und 5 Sekunden lang mit 10 $\mathrm{mg} / \mathrm{Hub}$.

In Abb. 4 sieht man die gemessenen Sensorsignale während des Versuchs am Motorprüfstand. In schwarz ist die Spannung des Sensors vor Diesel-Oxidations-Katalysator und in rot gestrichelt die Spannung des Sensors nach Diesel-Oxidations-Katalysator dargestellt. Zum Vergleich der beiden Sensoren wurde der Startwert auf Null gesetzt und somit eine Offsetkorrektur durchgeführt. Es ist deutlich zu erkennen, dass der Sensor nach Diesel-Oxidations-Katalysator eine geringeres Signal $\mathrm{zu}$ den Zeiten der Nacheinspritzungen zeigt als der Sensor vor Diesel-Oxidations-Katalysator, da einige Kohlenwasserstoffe bereits im DieselOxidations-Katalysator umgesetzt werden und somit nicht mehr zur exothermen Reaktion an der katalytischen Schicht des Sensors beitragen. Der Umsatz von Kohlenwasserstoffen und somit die Funktionalität des Diesel-Oxidations-Katalysators kann also sensorisch erfasst werden.

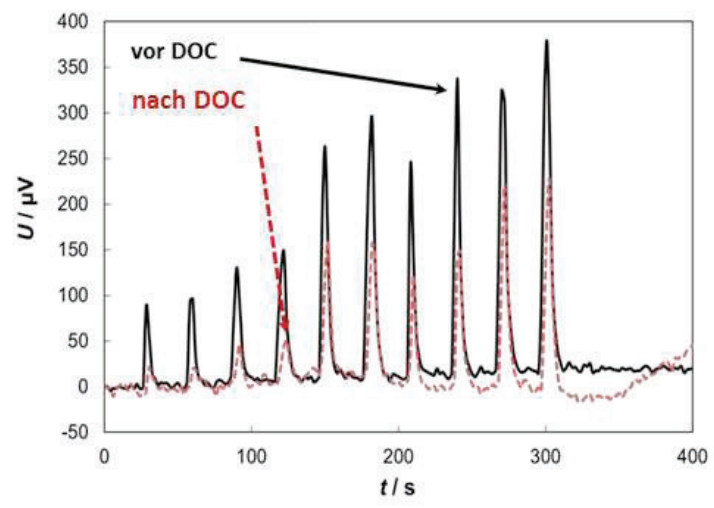

Abb. 4: Signale der Sensoren vor bzw. nach Diesel-Oxidations-Katalysator während den Nacheinspritzungen bei einer Motormessung. schwarz: gemessenes Signal vor dem Diesel-OxidationsKatalysator, rot und gestrichelt: gemessene Signale nach DieselOxidations-Katalysator.

\section{Fazit und Ausblick}

Die in Dickschichttechnik hergestellten thermoelektrischen Kohlenwasserstoffsensoren konnten erfolgreich getestet werden. Es hat sich gezeigt, dass die Sensoren mit einem 
stabilen und reproduzierbaren Signal auf Änderungen in der Kohlenwasserstoffkonzentration reagieren. Die Kennlinien entsprechen den theoretischen Erwartungen. Mithilfe der jeweiligen Seebeck-Koeffizienten der Materialpaarungen wurde deutlich, dass auf dem gleichen Substratmaterial auch die gleiche Temperaturdifferenz aufgrund der exothermen Reaktion gemessen wird.

Die Sensoren mit Pt|PtRh-Thermopaaren sind deutlich temperaturstabiler, so dass mit diesen Sensoren erste vielversprechende Ergebnisse im realen Abgas eines Motorprüfstandes durchgeführt werden konnten. Mit einem Sensor vor und einem Sensor nach Diesel-Oxidations-Katalysator konnte der Kohlenwasserstoffumsatz am Katalysator nachgewiesen werden.

Um die Empfindlichkeit noch weiter zu steigern, wird in folgenden Arbeiten die Anzahl an Thermoelementpaaren noch weiter erhöht werden.

\section{Danksagung}

Herzlicher Dank für die Förderung des Projekts geht an das Bayerische Staatsministerium für Wirtschaft und Medien, Energie und Technologie im Rahmen des Forschungsund Entwicklungsprogramms "Mikrosystemtechnik Bayern" (BAY174/002-1210-0003).

\section{Literaturnachweis}

[1] M. V. Twigg, Applied Catalysis B: Environmental, 70 (2007) 2-15, doi:10.1016/j.apcatb.2006.02.029

[2] P. L.T. Gabrielsson, Topics in Catalysis, 28 (2004) 177-184,

[3] Martyn V. Twigg et al., Platinum Metals Review, 53 (2009) 27-34, doi:10.1595/147106709×390977

[4] H.-O. Herrmann et al., Motortechnische Zeitschrift, 9 (2001) 652-690.

[5] Verordnung (EU) Nr. 459/2012, Emissionen von leichten Personenkraftwagen und Nutzfahrzeugen (Euro 6), Amtsblatt der Europäischen Union, 29.05.2012.

[6] J. Riegel et al., Solid State lonics, 152-153 (2002) 783-800, doi:10.1016/S01672738(02)00329-6

[7] R. Moos, International Journal of Applied Ceramic Technology, 2 (2005) 401-413, doi:10.1111/j.1744-7402.2005.02041.x

[8] L. F. Houlet et al., Sensors and Actuators B: Chemical, 130 (2008) 200-206, doi:10.1016/j.snb.2007.07.119

[9] S. Wiegärtner et al., Sensors and Actuators B: Chemical, 214 (2015), S. 234-240, doi:10.1016/j.snb.2015.02.083
[10] P. Gélin et al., Applied Catalysis B: environmental, 39 (2002) 1-37, doi: 10.1016/S0926-3373(02)00076-0 